\title{
Síntese e caracterização de uma nova meso-porfirina e derivados metalados
}

\section{Synthesis and characterization of a novel meso-porphyrin and its metallo derivatives}

\author{
Paulo Cesar Bega ${ }^{1}$; Jacqueline Aparecida Marques ${ }^{2 *}$
}

\begin{abstract}
Resumo
O interesse pela síntese de porfirinas e derivados vem se tornando cada vez mais crescente devido à grande eficiência catalítica de seus derivados metalados em reações de oxidação de hidrocarbonetos, à detecção de oxigênio, entre outras. Neste sentido foi realizado um estudo visando a síntese de uma nova porfirina, a 5,10,15,20-tetrakis(4-butóxi-3-metóxifenil)porfirina, e seus derivados metalados. A porfirina como base livre foi preparada à partir de um reagente acessível e de baixo custo, a vanilina. A alquilação da vanilina com $n$-bromobutano levou ao precursor sintético que foi então submetido às condições de Adler-Longo, na presença de pirrol e ácido propiônico, fornecendo a porfirina desejada. A porfirina purificada como base livre foi então submetida ao processo de metalação com sais de ferro (II) e manganês (II). Os novos compostos sintetizados foram caracterizados por espectroscopia de IV, UV-Vis, RMN e RPE.
\end{abstract}

Palavras-chave: Síntese. Porfirina apolar. Metalo porfirinas. Vanilina.

\begin{abstract}
There has been a growing interest in the properties of substituted meso-tetraarylporphyrins and metallo porphyrins as catalysts for oxidation of hydrocarbons, oxygen detection, among others. This work describes the synthesis of a new porphyrin, 5,10,15,20-tetrakis(4-butoxy-3-methoxyphenyl)porphyrin, and its metallo complexes. Herein it was used a readily available reactant, vanillin, as starting material which was submitted to alkylation with $n$-bromobutane affording the synthetic precursor. The desired porphyrin was obtained by reacting the $O$-alkylated aldehyde with pyrrole in the presence of propionic acid (Alder-Longo method). The purified porphyrin was then subjected to the metallation process using iron (II) and manganese (II) salts. The synthesized compounds were characterized by IR, UV-Vis, NMR and EPR spectroscopy
\end{abstract}

Keywords: Synthesis. Non polar porphyrin. Metallo porphyrins. Vanillin.

1 Graduado em Farmácia e Mestre em Química, Programa de Pós-Graduação em Química Aplicada, Universidade Estadual de Ponta Grossa.

2 Professora Associada do Departamento de Química, Universidade Estadual de Ponta Grossa, Paraná, Brasil. E-mail: jmarques@ uepg.br

* Autor para correspondência 


\section{Introdução}

As porfirinas formam uma importante classe de compostos com quatro unidades de pirrol (Figura 1) ligadas por pontes de $\mathrm{CH}$ (metino) e possuem um sistema eletrônico altamente conjugado de 22 elétrons $\pi$. Devido a esta extensa conjugação, as transições eletrônicas $\left(\pi \rightarrow \pi^{*}\right)$ encontram-se na faixa do visível e ultravioleta próximo conferindo sua coloração púrpura característica. Apresentam, portanto, um macrociclo central formado por 20 átomos de carbonos e 4 átomos de nitrogênio, chamado de porfina 1 (Figura 1) (MILGROM, 1997).

Figura 1 - Estrutura da porfina 1 e numeração sistemática (IUPAC).

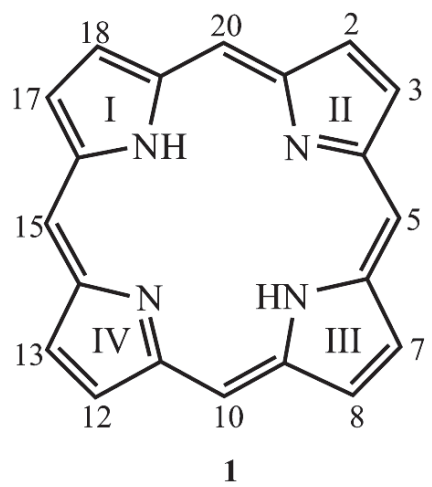

Fonte: Autor

Os anéis pirrólicos são numerados empregandose algarismos romanos de I a IV, os carbonos 5, 10,15 e 20 são denominados de posições meso, os carbonos 2, 3, 7, 8, 12, 13, 17 e 18 são denominados de posições $\beta$-pirrólicas e os carbonos restantes 1 , $4,6,9,11,14,16$ e 19 são as posições $\alpha$ (Figura 1) (MILGROM, 1997).

O macrociclo possui dois átomos de nitrogênios imínicos capazes de aceitar prótons (22 e 24 dos anéis II e IV) e dois nitrogênios amínicos capazes de ceder prótons (21 e 23 dos anéis I e III). A substituição dos dois átomos de hidrogênio centrais ligados ao nitrogênio do anel na molécula da porfirina na forma de base livre por um cátion metálico leva a formação de uma porfirina metalada. $\mathrm{O}$ anel central da porfirina possui $70 \mathrm{pm}$ e o íon de ferro (III) possui $65 \mathrm{pm}$, um valor considerado ideal para a formação de metaloporfirinas, sendo que as metaloporfirinas com ferro são facilmente encontradas na natureza (KAIM; SCHWEDERSKI, 1994).

Entre as diversas aplicações das porfirinas, se destaca sua importância na medicina, pois possuem alta afinidade por tecidos tumorais e alta atividade fotodinâmica. Estas características as tornam drogas eficazes para diagnóstico e tratamento de câncer através de Terapia Fotodinâmica (TFD). Além da atividade fotodinâmica, alguns derivados metalados das porfirinas são capazes de promover oxidação catalítica e seletiva de alcanos (DOLPHIN; TRAYLOR; XIE, 1997; SIMPLICIO; MAIONCHI; HIOKA, 2002).

Areação de oxidação catalítica de hidrocarbonetos é alvo constante de estudo de muitos pesquisadores. A oxidação de hidrocarbonetos saturados a álcoois ou cetonas é de difícil controle, pois estes produtos se oxidam com mais facilidade do que os hidrocarbonetos (DOLPHIN; TRAYLOR; XIE, 1997).

Neste sentido, há um grande interesse pela síntese de porfirinas e derivados, os quais já demonstraram uma grande eficiência em reações de oxidação de hidrocarbonetos. Alguns tipos de porfirinas metaladas podem ser usadas como catalisadores em diversas reações, como a hidroxilação do cicloexano, ciclopropanações, reações de epoxidação, detecção de oxigênio e modelos para eficiência catalítica do citocromo P-450, entre outras (DOLPHIN; TRAYLOR; XIE, 1997; SCHIAVON et al., 2000).

As porfirinas também têm sido usadas como sistemas modelos para entender o processo de fotossíntese e, assim, buscar sistemas para aproveitamento da energia solar e novos materiais ópticos estão sendo desenvolvidos baseados no planejamento racional a na síntese de modelos biomiméticos contendo porfirinas (metaloporfirinas). Alguns fatores tornam as 
porfirinas cromóforos vantajosos sobre outros sistemas doadores, tais como um grande número de vias sintéticas, altos valores de coeficiente de absortividade molar e rendimentos quânticos de emissão bastante significativos. Por estes motivos, existe um grande número de pesquisas usando sistemas porfirínicos como doadores-receptores em estudos de transferência de elétrons e energia induzidos fotoquimicamente (NERI et al., 2002).

As porfirinas na forma de base livre não apresentam atividade catalítica na oxidação de substratos orgânicos. As porfirinas substituídas com grupos fenila nas posições meso do anel representam a classe de porfirinas denominadas de primeira geração, por terem sido as primeiras porfirinas estudadas com fins catalíticos e são também denominadas de meso-substituídas (DOLPHIN; TRAYLOR; XIE, 1997)

As porfirinas meso-substituídas são preferencialmente preparadas a partir de reações de condensação entre pirróis e aldeídos. O maior avanço nesse processo de condensação ocorreu com o trabalho de Adler et al., em 1964, que mostrou que o rendimento da reação poderia ser significativamente aumentado se a reação fosse realizada em meio ácido e em recipiente aberto. O procedimento consistia em reagir o benzaldeído com pirrol recémdestilado na proporção de $1: 1$, em refluxo de ácido propiônico, a $141{ }^{\circ} \mathrm{C}$, por 30 minutos ao ar livre (condições aeróbicas). O rendimento aumentou de $3-5 \%$ para $20 \%$ e o produto obtido por cristalização sob resfriamento, tornando a purificação mais simples (ADLER et al., 1967; KIM; LEONARD; LONGO, 1972; SMITH, 2000).

A partir de então, diversas adaptações foram sugeridas para este método, com a intenção de aumentar o rendimento e facilitar a purificação, diminuindo a quantidade de subprodutos. Dentre eles destacam-se as modificações propostas por Lindsey e por Gonsalves (GONSALVES; VAREJÃO; PEREIRA, 1991; JOHNSTONE et al., 1996; LINDSEY et al., 1987).
Embora, para tais métodos, em geral, os rendimentos sejam da ordem de $30-40 \%$, a aplicação é destinada a síntese de porfirinas substituídas cuja rota não é adequada via Adler-Longo, sendo que, por exemplo, a principal desvantagem do método de Lindsey é a necessidade de alta diluição (10-2 mol. $\left.L^{-1}\right)$.

Portanto, neste trabalho o método utilizado no preparo da TBMPP 5 foi o de Adler-Longo, por ser experimentalmente mais simples e por proporcionar a obtenção da porfirina em escala multigramas.

\section{Parte experimental}

\section{Generalidades}

Os espectros de absorção eletrônica no UVVis foram obtidos em um espectrofotômetro HP8452A e células espectrofotométricas de quartzo, com caminho óptico de $1,0 \mathrm{~cm}$. Os espectros vibracionais na região do IV foram registrados em um espectrofotômetro BOMEM, série MB e BIORAD, FTS-3500 GX, com transformada de Fourier e calibração interna. As amostras foram preparadas na forma de pastilhas de $\mathrm{KBr}$ e as absorções estão expressas em número de onda $\left(\mathrm{cm}^{-1}\right)$.

Os espectros de RPE foram obtidos em um espectrômetro Bruker ESP 300 E na banda-X (aproximadamente 9,5 GHz), a $77 \mathrm{~K}$ utilizando-se $\mathrm{N}_{2}$ líquido e amostras sólidas pulverizadas em tubo de quartzo.

Os espectros de RMN foram registrados em um espectrômetro Bruker ARX-400 operando a $400 \mathrm{MHz}$ para ${ }^{1} \mathrm{H}$ e $100 \mathrm{MHz}$ para ${ }^{13} \mathrm{C}$, utilizandose clorofórmio deuterado como solvente. Todos os espectros foram registrados utilizando-se tetrametilsilano como padrão de referência interno. Os deslocamentos químicos ( $\delta$ ) estão expressos em ppm e as constantes de acoplamento $(J)$ em Hertz $(\mathrm{Hz})$. Para indicar a multiplicidade dos sinais foram adotadas as seguintes abreviações: s (singleto), sl (singleto largo), d (dubleto), $\mathrm{t}$ (tripleto), qt (quintupleto) e st (sexteto). 
Nas separações feitas por cromatografia em coluna utilizou-se sílica gel 60 70-230 mesh ASTM Merck. As análises cromatográficas em camada delgada (CCD) foram efetuadas utilizando-se placas cromatográficas de sílica gel comerciais da Merck (Sílica Gel 60 F254). Estas foram reveladas sob luz ultravioleta $(\lambda=254 \mathrm{~nm})$ ou iodo molecular.

Todas as reações foram monitoradas por CCD e/ou UV-Vis. Os solventes foram removidos em evaporador rotatório (rotoevaporador) operando à pressão reduzida ( $\approx 30-40 \mathrm{mmHg})$.

Neste trabalho foram utilizados os seguintes compostos: acetona, $n$-hexano, $n$-bromobutano, acetato de etila (AcOEt), cloreto de ferro (II) trihidratado, diclorometano (DCM), carbonato de potássio anidro, hidróxido de sódio, metanol, $N, N$ dimetilformamida (DMF), cloreto de manganês (II) tetra-hidratado, água destilada e água deionizada. As peneiras moleculares ( 3 e 4 A $)$ utilizadas para secagem de solventes foram previamente ativadas em mufla a $300{ }^{\circ} \mathrm{C}$, por um período de 3 $\mathrm{h}$, e mantidas em dessecador à vácuo até o uso. A acetona, o DMF e o $n$-bromobutano $(n$ - $\mathrm{BuBr})$ foram tratados de acordo com procedimento descrito em literatura (ARMAREGO; PERRIN, 2000).

Todas as reações de metalação foram realizadas sob atmosfera inerte (argônio). Foram utilizados DCM como eluente para CCD e DMF como solvente para UV-Vis. Para a medida do coeficiente de absortividade molar ( $\varepsilon$ ) preparou-se uma solução estoque da porfirina utilizando-se DMF como solvente. A partir dos dados de absorbância obtidos, calculou-se $o$ e da porfirina em determinados comprimentos de onda, utilizando-se a Lei de BeerLambert.

Os procedimentos descritos se referem aos melhores resultados obtidos em termos de rendimento.

\section{Síntese da 5,10,15,20-tetrakis(4-butóxi-3- metóxifenil)porfirina, TBMPP 5.}

Em um balão fundo redondo de $500 \mathrm{~mL}$ foram misturados vanilina $2(16,0 \mathrm{~g} ; 0,105 \mathrm{mols})$, carbonato de potássio anidro $(15,0 \mathrm{~g} ; 0,108 \mathrm{~mol})$, brometo de $n$-butila (16,0 g; 0,116 mols) e acetona anidra (107 $\mathrm{mL}$ ). A mistura foi deixada em refluxo sob agitação a $60{ }^{\circ} \mathrm{C}$ por 22 horas. Na CCD foi utilizado como eluente uma mistura de AcOEt e $n$-hexano 6:4 (v/v).

O solvente da reação foi removido e ao resíduo foi adicionado éter etílico. Transferiu-se a mistura para um funil de separação e a fase etérea foi lavada com água destilada e depois com uma solução de $\mathrm{NaOH}$ 10\%. A fase orgânica foi seca com sulfato de sódio anidro, filtrada e concentrada em rotoevaporador. Obteve-se 12,6 g (57,5\%) do $n$-butil metil aldeído 3 como um óleo de coloração levemente amarelada. O aldeído 3 foi utilizado na etapa seguinte sem posterior purificação.

Em um balão de fundo redondo de $500 \mathrm{~mL}$, foram misturados ácido propiônico $(170 \mathrm{~mL})$ e 3-metóxi-4-butóxi benzaldeído 3 (14,9 g; 0,072 mols). Aqueceu-se até refluxo sob agitação magnética, e então, adicionou-se pirrol $(5,0 \mathrm{~mL}$; $4,83 \mathrm{~g} ; 0,072 \mathrm{mols}$ ). Após 30 minutos o solvente foi removido por destilação e a porfirina foi purificada por cromatografia em coluna utilizando-se como eluente uma mistura de AcOEt e $n$-hexano 8:2 $(\mathrm{v} / \mathrm{v})$. O solvente foi removido em rotoevaporador fornecendo $1,5 \mathrm{~g}(8,3 \%)$ da porfirina 5 como um sólido de coloração púrpura.

\section{Síntese do acetato de $[5,10,15,20$-tetrakis(4- butóxi-3-metóxifenilporfirina) manganês (III)], Mn TBMPP 6.}

Em um balão de fundo redondo de três bocas de $125 \mathrm{~mL}$ foi adicionado DMF $(15 \mathrm{~mL})$ recémdestilado. O sistema foi desaerado com argônio. Aqueceu-se até refluxo sob agitação magnética e adicionou-se a porfirina TBMPP 5 (100 mg; 9,77. $10^{-5}$ mols) e de cloreto de manganês $(192 \mathrm{mg}$; 
$9,71 \cdot 10^{-4}$ mols). A reação foi mantida sob refluxo por $7 \mathrm{~h}$.

Ao final desse tempo, a mistura reacional foi resfriada até t.a. e acrescentou-se metanol. Transferiu-se a mistura para um balão de $500 \mathrm{~mL}$ e concentrou-se em rotoevaporador. Para remoção do DMF residual adicionou-se tolueno e concentrouse novamente em rotoevaporador. Ao resíduo foi adicionado DCM e transferiu-se a mistura para um funil de separação e a fase orgânica foi lavada com água destilada. $\mathrm{O}$ solvente foi removido em rotoevaporador e obteve-se $64,8 \mathrm{mg}(59,7 \%)$ da porfirina metalada 6 como um sólido de coloração esverdeada.

Síntese do cloreto de [5,10,15,20-tetrakis(4butóxi-3-metóxifenilporfirina) ferro (III)], FeTBMPP 7.

Em um balão de fundo redondo de três bocas de $125 \mathrm{~mL}$ foi adicionado DMF $(50 \mathrm{~mL})$ recémdestilado. O sistema foi desaerado com argônio. Aqueceu-se até refluxo sob agitação magnética e adicionou-se a porfirina TBMPP 5 (200 mg; 0,195 mmol) e cloreto de ferro (II) (386 mg; 2,14 mmol) tri-hidratado. A reação foi mantida sob refluxo por $5 \mathrm{~h}$.
Ao final desse tempo, a mistura reacional foi resfriada até t.a. e acrescentou-se metanol. Transferiu-se a mistura para um balão de $500 \mathrm{~mL}$ e concentrou-se em rotoevaporador. Para remoção do DMF residual adicionou-se tolueno e concentrouse novamente em rotoevaporador. Ao resíduo foi adicionado DCM e transferiu-se a mistura para um funil de separação e a fase orgânica foi lavada com água destilada. $\mathrm{O}$ solvente foi removido em rotoevaporador e obteve-se $131 \mathrm{mg}(60,4 \%)$ da porfirina metalada 7 como um sólido de coloração marrom avermelhada.

\section{Resultados e discussão}

O aldeído precursor, o 3-metóxi-4butóxibenzaldeído 3 , foi obtido num rendimento de $57,5 \%$ por meio da alquilação $\left(\mathrm{S}_{\mathrm{N}} 2\right)$ do íon fenóxido gerado in situ a partir da vanilina 2 (Figura 2).

Para a síntese da TBMPP 5, foi escolhido o método Adler-Longo. A reação ocorreu em 30 minutos (ca.), com concomitante oxidação in situ do porfirinogênio 4 , uma vez que a reação é realizada em recipiente aberto, tendo o $\mathrm{O}_{2}$ atmosférico como oxidante (Figura 2).

Figura 2 - Síntese da TBMPP 5 e metalo porfirinas 6 e 7.

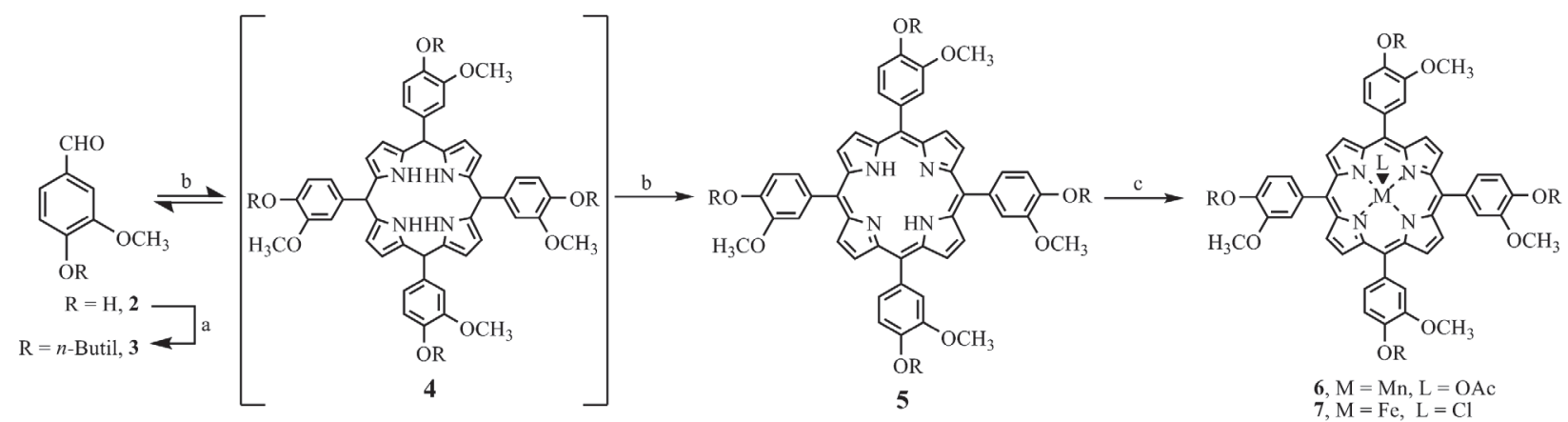

Reagentes e condições: (a) $\mathrm{K}_{2} \mathrm{CO}_{3}, n$ - $\mathrm{BuBr}$, acetona, refluxo, 57,5\%; (b) pirrol, ácido propiônico, $\mathrm{O}_{2}$, refluxo, 8,3\%; (c) $\mathrm{MnCl}_{2} \cdot 4 \mathrm{H}_{2} \mathrm{O}$, DMF, refluxo, 59,7\%; ou, $\mathrm{FeCl}_{2} .3 \mathrm{H}_{2} \mathrm{O}$, DMF, refluxo, $60,4 \%$.

Fonte: Autor 
Após a síntese e purificação, a base livre TBMPP 5 foi submetida à análise por UV-Vis (Figura 3). No espectro eletrônico da solução desta porfirina em DMF observa-se a banda Soret em $426 \mathrm{~nm}$ e as quatro bandas Q (IV, III, II e I) em 518, 556, 594 e 652 nm, respectivamente. A banda Soret e as bandas Q estão dentro da faixa de absorção características, ou seja, entre 410 e $450 \mathrm{~nm}$ para a banda Soret e entre 500 e $650 \mathrm{~nm}$ para as bandas Q (KAIN; SCHWEDERSKI, 1994; KIM; LEONARD; LONGO, 1972; NIAN; MIN; KONG, 1990). O valor encontrado para o coeficiente de absortividade molar $(\varepsilon)$ da banda Soret da TBMPP 5 foi de 3,78.10 L. $\mathrm{mol}^{-1} \mathrm{~cm}^{-1}$.

Figura 3 - Espectro de UV-VIS da solução da porfirina base livre TBMPP 5 em DMF.

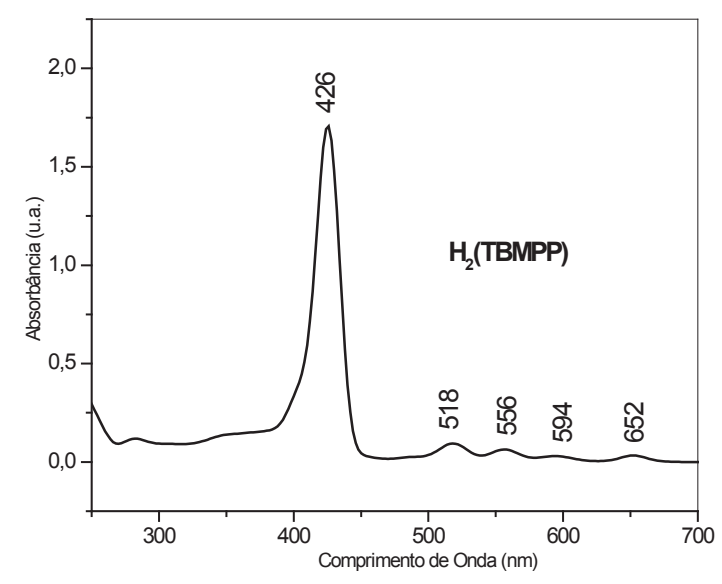

Fonte: Dados do autor.
A Figura 4 apresenta o espectro de IV da TBMPP 5. A análise deste espectro permite uma caracterização da referida porfirina, por meio da observação de alguns picos de absorção característicos. Na Tabela 1 estão as principais frequências de absorção na região do infravermelho correspondentes a porfirina TBMPP 5.

O espectro de RMN de ${ }^{1} \mathrm{H}$ (Figura 5) da TBMPP 5 possui os sinais característicos dos oito hidrogênios $\beta$-pirrólicos.

Tabela 1 - Principais vibrações da TBMPP 5.

\begin{tabular}{ll}
\hline \multicolumn{1}{c}{ Absorção(ões) $\left(\mathrm{cm}^{-1}\right)$} & \multicolumn{1}{c}{ Atribuição } \\
\hline 3.289 & $\vee \mathrm{N}-\mathrm{H}$ \\
$2.930 ; 2.843$ & $\vee \mathrm{C}-\mathrm{H}(n$-butil) \\
$1.555 ; 1.483 ; 1.443$ & $\vee \mathrm{C}=\mathrm{C}$ (aril) \\
1.227 & $v_{\text {as }} \mathrm{C}-\mathrm{O}-\mathrm{C}$ (alquil aril éter) \\
$1.111 ; 1.011$ & $v_{\mathrm{s}} \mathrm{C}-\mathrm{O}-\mathrm{C}$ (alquil aril éter) \\
775 & $\delta \mathrm{C}-\mathrm{H}$ (aril $p$-di-substituído) \\
712 & $\delta \mathrm{C}-\mathrm{H}$ (pirrólico) \\
\hline$\delta=$ deformação angular; $v=$ deformação axial; as = assimétrica; \\
s= simétrica \\
Fonte: Dados do autor.
\end{tabular}

Figura 4 - Espectro de IV da TBMPP 5.

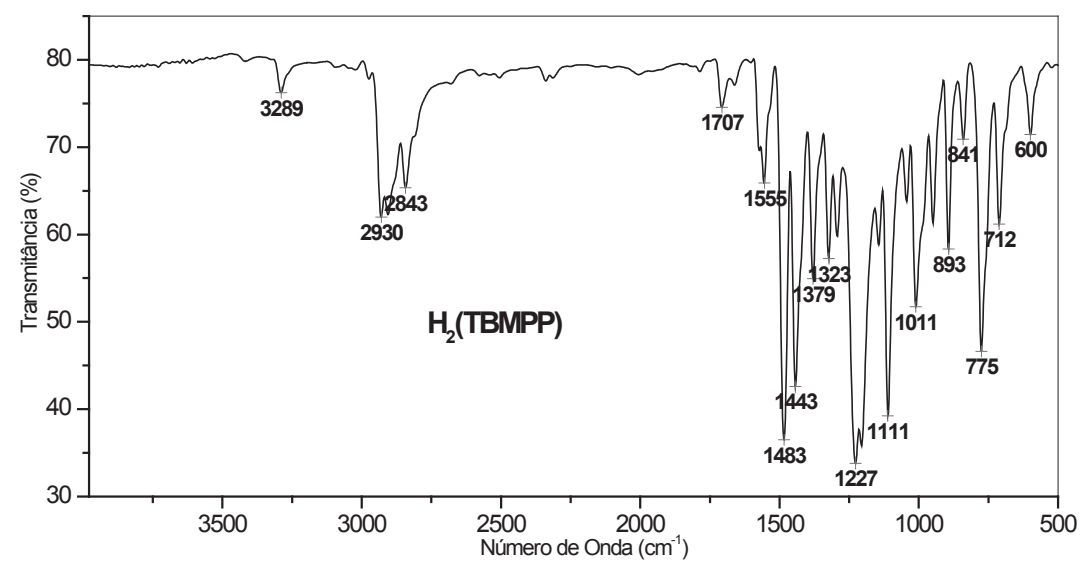

Fonte: Dados do autor. 
Figura 5 - Espectro de RMN de ${ }^{1} \mathrm{H}$ da TBMPP 5.

(d)

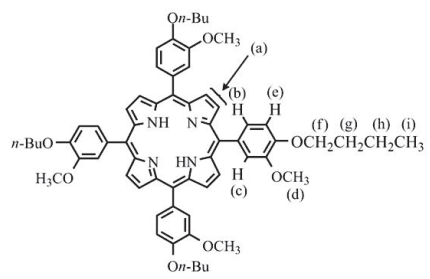

(a)

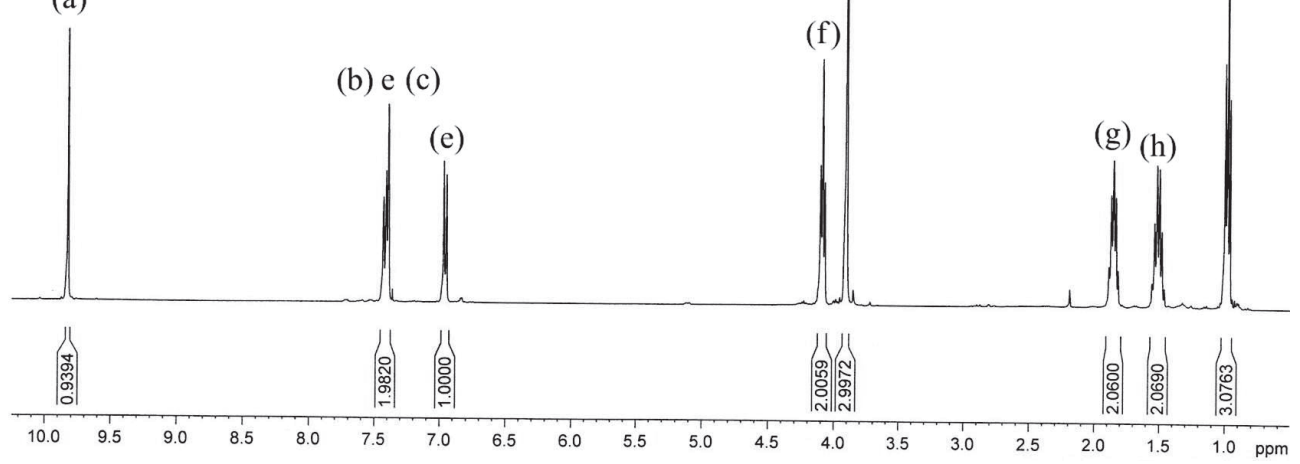

Fonte: Dados do autor.

$\mathrm{H}(\mathrm{a})$ em $\delta$ 9,83 um dubleto atribuído aos quatro hidrogênios $\mathrm{H}(\mathrm{b})$ em $\delta$ 7,42; um singleto largo atribuído aos quatro hidrogênios $\mathrm{H}(\mathrm{c})$ em $\delta$ 7,39; um dubleto em $\delta$ 6,96 atribuído aos quatro hidrogênios $\mathrm{H}(\mathrm{e})$; um tripleto em $\delta 4,09$ atribuído aos oito hidrogênios metilênicos $\mathrm{H}(\mathrm{f})$; um singleto em $\delta$ 3,91 atribuído aos doze hidrogênios $\mathrm{H}(\mathrm{d})$ das metoxilas; um quintupleto em $\delta 1,86$ e um sexteto em $\delta 1,51$, ambos atribuídos, respectivamente, aos oito hidrogênios $H(g)$ e aos oito hidrogênios $H(h)$ dos grupos metilenos $\beta$ e $\gamma$ ao átomo de oxigênio e um tripleto em $\delta 0,99$ correspondente aos doze hidrogênios $\mathrm{H}(\mathrm{i})$ dos grupos metilas. Os dois hidrogênios pirrólicos N-H aparecem como um singleto largo em $-2,72$, característico de porfirinas na forma de base livre. Os valores dos deslocamentos químicos e as multiplicidades estão de acordo com os dados de literatura (GONSALVES; VAREJÃO; PEREIRA, 1991; LINDSEY et al., 1987; SMITH, 2000).

Tabela 2 - Atribuições dos sinais de RMN de ${ }^{1} \mathrm{H}$ da TBMPP 5.

\begin{tabular}{ccccc}
\hline Hidrogênio & $\delta(\mathrm{ppm})$ & Multiplicidade & $\mathrm{n}^{0}$. hidrogênios & $\mathrm{J}(\mathrm{Hz})$ \\
\hline (a) & 9,82 & $\mathrm{~s}$ & $8 \mathrm{H}$ & \\
(b) & 7,42 & $\mathrm{~d}$ & $4 \mathrm{H}$ & 8,4 \\
(c) & 7,39 & $\mathrm{sl}$ & $4 \mathrm{H}$ & \\
(e) & 6,96 & $\mathrm{~d}$ & $4 \mathrm{H}$ & 8,4 \\
(f) & 4,09 & $\mathrm{t}$ & $8 \mathrm{H}$ & \\
(d) & 3,91 & $\mathrm{~s}$ & $12 \mathrm{H}$ & 7,6 \\
(g) & 1,86 & $\mathrm{qt}$ & $8 \mathrm{H}$ & 7,6 \\
(h) & 1,51 & $\mathrm{st}$ & $8 \mathrm{H}$ & \\
(i) & 0,99 & $\mathrm{t}$ & $12 \mathrm{H}$ & \\
\hline
\end{tabular}

Fonte: Dados do autor. 
A Figura 6 apresenta os sinais de $\mathrm{RMN}$ de ${ }^{13} \mathrm{C}$ da TBMPP 5 e, na Tabela 3, estão as atribuições dos sinais observados utilizando-se também o espectro de DEPT-135. Em relação espectro de RMN de ${ }^{13} \mathrm{C}$ destaca-se a ausência do sinal do carbono $\alpha$-pirrólico (C-14) que por ser extremamente alargado, torna-se difícil de ser detectado para a grande maioria das porfirinas tetra-substituídas simétricas. Já o carbono $\beta$-pirrólico (C-4) aparece como um pico alargado. Os valores de deslocamentos químicos para os treze carbonos detectados (dentre os quatorze existentes) estão de acordo com os da literatura (UCOSKI et al., 2011; SHAW et al., 2001).

Figura 6 - Espectro de RMN de ${ }^{13} \mathrm{C}$ da TBMPP 5.

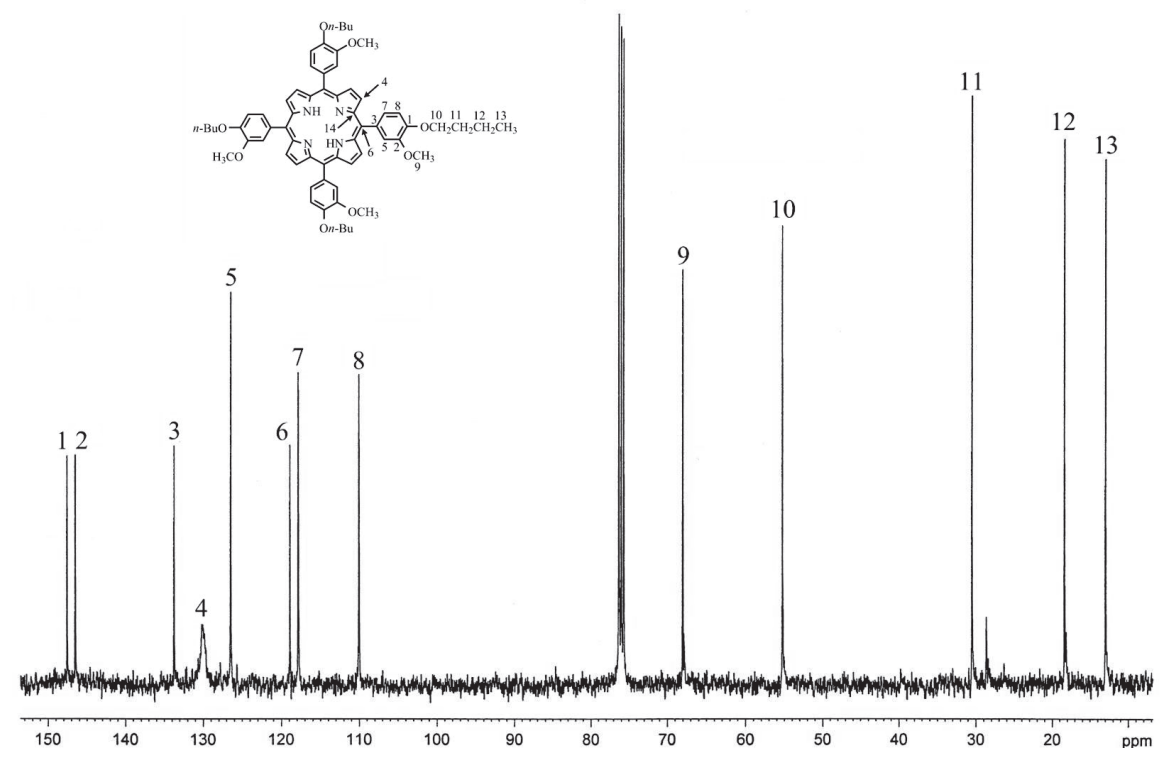

Fonte: Dados do autor.

Tabela 3. Atribuições dos sinais de $\mathrm{RMN}$ de ${ }^{13} \mathrm{C}$ da TBMPP 5.

\begin{tabular}{ccc}
\hline Carbono & $\delta(\mathrm{ppm})$ & DEPT-135 \\
\hline 1 & 147,54 & --- \\
2 & 146,46 & --- \\
3 & 133,64 & --- \\
4 & 130,09 & $\mathrm{CH}$ (alargado) \\
5 & 126,54 & $\mathrm{CH}$ (positivo) \\
6 & 118,90 & --- \\
7 & 117,74 & $\mathrm{CH}$ (positivo) \\
8 & 110,02 & $\mathrm{CH}$ (positivo) \\
9 & 68,10 & $\mathrm{CH}_{2}$ (negativo) \\
10 & 55,42 & $\mathrm{CH}_{3}$ (positivo) \\
11 & 30,52 & $\mathrm{CH}_{2}$ (negativo) \\
12 & 18,39 & $\mathrm{CH}_{2}$ (negativo) \\
13 & 12,98 & $\mathrm{CH}_{3}$ (positivo) \\
\hline
\end{tabular}

A porfirina 5 foi metalada com os sais doadores de metais (excesso de $c a .10$ vezes) utilizando-se como solvente a $N, N$-dimetilformamida (DMF) (FRIEDERMANN et al., 2006). Para a reação de metalação foi necessário encontrar um solvente adequado que solubilizasse a porfirina, TBMPP 5 e os sais dos íons metálicos utilizados. A solubilidade de porfirinas na forma de base livre em um número maior de solventes orgânicos, tanto polares quanto apolares é uma característica desejável. Assim, inicialmente, foram realizados testes de solubilidade da TBMPP 5 em vários solventes a $25^{\circ} \mathrm{C}$ (Tabela 4).

Fonte: Dados do autor. 
Tabela 4 - Testes de solubilidade da TBMPP 5.

\begin{tabular}{lc}
\hline \multicolumn{1}{c}{ Solvente } & Solubilidade \\
\hline Diclorometano & Solúvel \\
Acetonitrila & Pouco solúvel \\
$N, N$-Dimetilformamida & Solúvel \\
Dimetilsulfóxido & Solúvel \\
Tolueno & Solúvel \\
Etanol & Pouco solúvel \\
Metanol & Pouco solúvel \\
Ácido acético & Solúvel \\
Clorofórmio & Solúvel \\
Água destilada & Pouco solúvel \\
Acetona & Solúvel \\
Tetraidrofurano & Solúvel \\
Acetato de etila & Solúvel \\
\hline
\end{tabular}

Fonte: Dados do autor.
Dentre os solventes em que a porfirina TBMPP 5 foi solúvel, o solvente escolhido foi DMF pois verificou-se que os sais de ferro e manganês também possuíam solubilidade satisfatória nesse solvente na temperatura de reação $\left(153^{\circ} \mathrm{C}\right)$. O DMF é fracamente coordenante e a temperatura elevada torna mais rápida a inserção do íon metálico.

Quando a porfirina é metalada, em geral, ocorre um deslocamento da banda Soret (B) para uma região de maior energia e as bandas $\mathrm{Q}$ são reduzidas a duas, Q $(1,0)$ e Q $(0,0)$, entre 500 e $700 \mathrm{~nm}$ (BOUCHER, 1970; FRIEDERMANN et al., 2006; GOUTERMAN, 1961).

Figura 7 - Espectros de UV-Vis das metaloporfirinas de manganês (a) MnTBMPP 6; e de ferro (b) FeTBMPP 7.

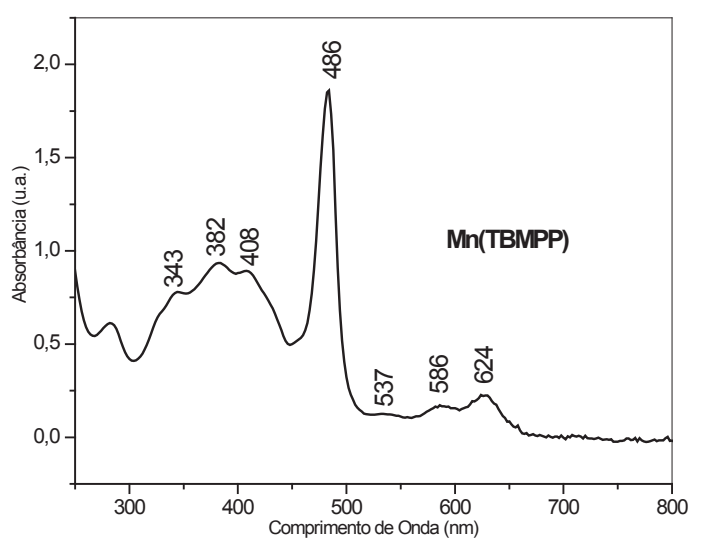

(a)

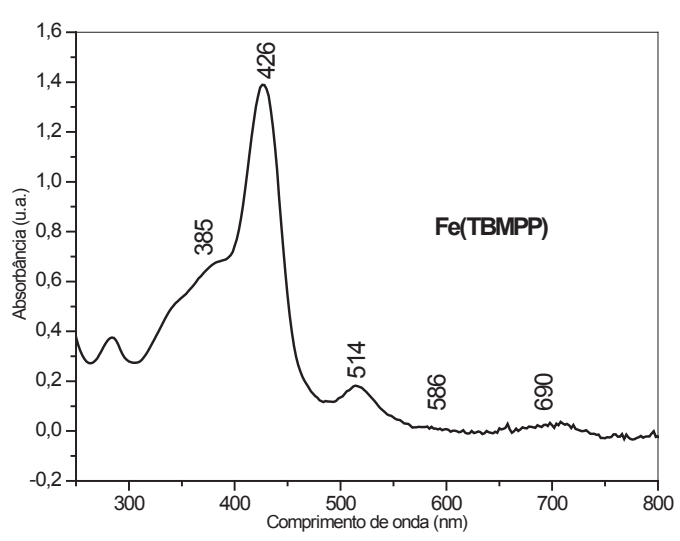

(b)

Fonte: Dados do autor.

A análise dos espectros (Figura 7) permite a comparação dos valores encontrados com aqueles descritos na literatura para porfirinas similares. Observa-se que as metaloporfirinas tiveram suas bandas Q reduzidas a três, mas apenas a MnTBMPP 6 apresentou deslocamento significativo da banda Soret para maior comprimento de onda (menor energia), de 426 para $486 \mathrm{~nm}$, característico de porfirinas de manganês (III). Para as demais bandas da MnTBMPP 6 observou-se os valores de 537, 586 e $624 \mathrm{~nm}$ caracterizando o processo de metalação (NIAN et al., 1990). As bandas em 343, 382 e $408 \mathrm{~nm}$ são atribuídas as transições eletrônicas de transferência de carga metal-porfirina, $\mathrm{a}_{1 \mathrm{u}}(\pi)$, $\mathrm{a}_{2 \mathrm{u}}(\pi) \rightarrow \mathrm{e}_{\mathrm{g}}(\pi *)$ (Figura 7a) (BOUCHER, 1970; FRIEDERMANN et al., 2006).

A Figura $7 \mathrm{~b}$ mostra o espectro eletrônico da porfirina de ferro (III) 7 onde são observadas 
as bandas Q em 514, 586 e $690 \mathrm{~nm}$, típicas do monômero. A banda em $385 \mathrm{~nm}$ juntamente com a banda em $514 \mathrm{~nm}$ pode ser atribuída a formação de uma espécie pentacoordenada tipica da coordenação do ligante cloro a FeTBMPP (PRADO-MANSO et al., 1999; NIAN; MIN; KONG, 1990).

As metaloporfirinas também foram caracterizadas por espectroscopia na região do IV e apresentam os picos de absorção característicos muito semelhantes com os picos da porfirina base livre TBMPP 5 (Tabela 2) à exceção do pico correspondente a deformação axial de N-H, ausente nas metaloporfirinas. A Figura 8 mostra os espectros de IV obtidos da MnTBMPP 6 e FeTBMPP 7, e as Tabelas 5 e 6 apresentam os principais picos de absorção das metaloporfirinas e as deformações correspondentes.

Figura 8 - Espectros de IV das metaloporfirinas de (a) MnTBMPP 6; e (b) FeTBMPP 7.

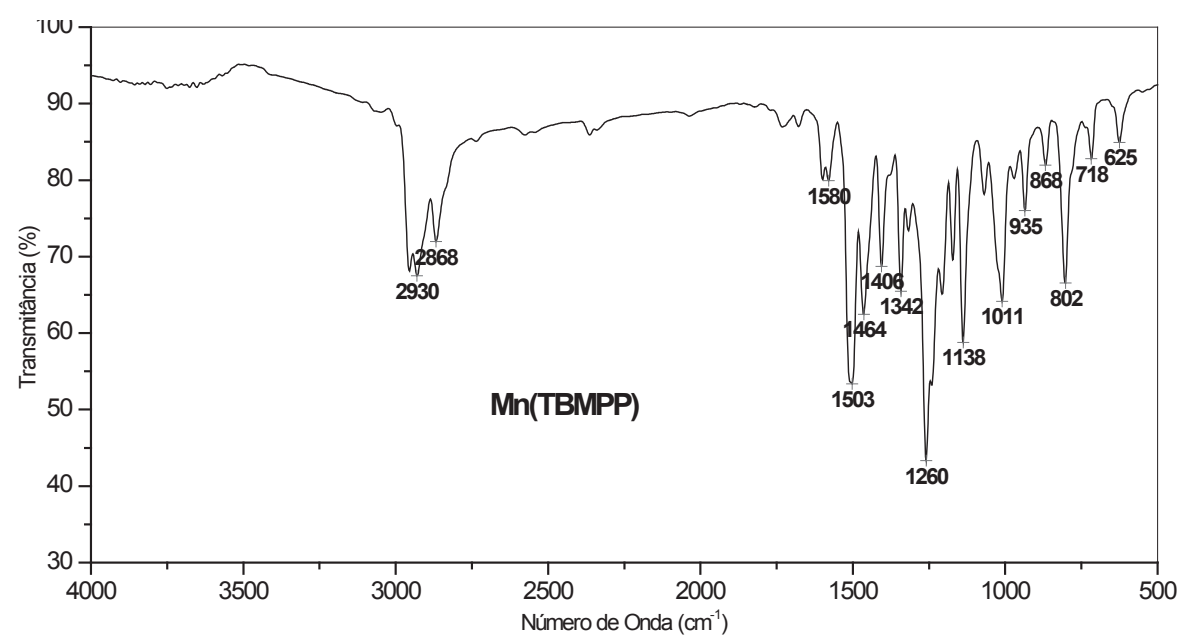

(a)

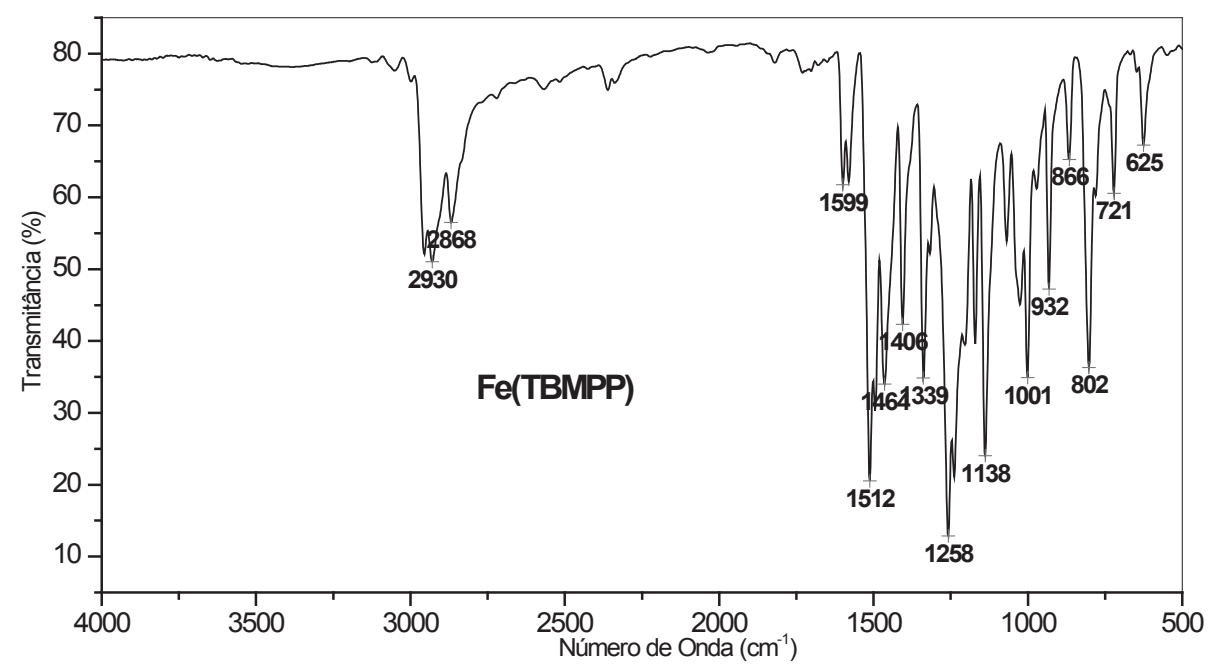

(b)

Fonte: Dados do autor. 
Tabela 5 - Principais vibrações da MnTBMPP 6.

\begin{tabular}{ll}
\hline \multicolumn{1}{c}{ Absorção(ões) $\left(\mathrm{cm}^{-1}\right)$} & \multicolumn{1}{c}{ Atribuição } \\
\hline $2.930 ; 2.868$ & $\vee \mathrm{C}-\mathrm{H}(n$-butil) \\
$1.580 ; 1.503 ; 1.464$ & $\vee \mathrm{C}=\mathrm{C}$ (aril) \\
1.260 & $v_{\text {as }} \mathrm{C}-\mathrm{O}-\mathrm{C}$ (alquil aril éter) \\
$1.138 ; 1.011$ & $v_{\mathrm{s}} \mathrm{C}-\mathrm{O}-\mathrm{C}$ (alquil aril éter) \\
802 & $\delta \mathrm{C}-\mathrm{H}$ (aril $p$-di-substituído) \\
718 & $\delta \mathrm{C}-\mathrm{H}$ (pirrólico) \\
\hline
\end{tabular}

$\delta=$ deformação angular; $v=$ deformação axial; as = assimétrica; $\mathrm{s}=$ simétrica

Fonte: Dados do autor.

Tabela 6 - Principais vibrações da FeTBMPP 7.

\begin{tabular}{ll}
\hline \multicolumn{1}{c}{ Absorção(ões) $\left(\mathrm{cm}^{-1}\right)$} & \multicolumn{1}{c}{ Atribuição } \\
\hline $2.930 ; 2.868$ & $v \mathrm{C}-\mathrm{H}(n$-butil) \\
$1.599 ; 1.512 ; 1.464$ & $v \mathrm{C}=\mathrm{C}$ (aril) \\
1.258 & $v_{\text {as }} \mathrm{C}-\mathrm{O}-\mathrm{C}$ (alquil aril éter) \\
$1.138 ; 1.001$ & $v_{\mathrm{s}} \mathrm{C}-\mathrm{O}-\mathrm{C}$ (alquil aril éter) \\
802 & $\delta \mathrm{C}-\mathrm{H}$ (aril $p$-di-substituído) \\
721 & $\delta \mathrm{C}-\mathrm{H}$ (pirrólico) \\
\hline
\end{tabular}

$\delta=$ deformação angular; $v=$ deformação axial; as = assimétrica; $\mathrm{s}=$ simétrica

Fonte: Dados do autor.

As metaloporfirinas foram caracterizadas por meio da técnica de RPE (Figura 9) com o objetivo de se confirmar o estado de oxidação dos metais após a etapa de metalação.

Modificações estruturais efetuadas nas porfirinas podem estabilizar estados diferentes de oxidação para o manganês, como: Mn (II), Mn (III) e Mn (IV). Os íons Mn (II) possuem configuração eletrônica $d 5$ com $\mathrm{S}=5 / 2$ e o $\mathrm{Mn}$ (IV) com configuração $d 3$, tem $\mathrm{S}=3 / 2$ em spin alto e $\mathrm{S}=1 / 2$ em spin baixo. $\mathrm{O}$ íon Mn (III) com configuração $d 4$ e $S=2$ possui 4 elétrons desemparelhados e exibe uma pronunciada distorção Jahn-Teller, que resulta em um substancial acoplamento spin-órbita. Por este motivo o íon Mn (III) não apresenta sinal em RPE. Portanto, a ausência de sinal de RPE (espectro silencioso) no espectro da [Mn(III)(TBMPP) $]^{+}$, sugere que o estado de oxidação do manganês neste complexo seja +3 (Figura 9a). Os complexos de ferro em sistemas porfirínicos são, frequentemente, pentacoordenados com o íon Fe (III), localizado um pouco acima do plano porfirínico. A Figura 9b mostra o espectro da porfirina de ferro, FeTBMPP 7, com um sinal em g $=5,3$, característico de Fe(III) spin alto em simetria axial (BOUCHER, 1970; FRIEDERMANN et al., 2006; KAIN, SCHWEDERSKI, 1994; UCOSKI et al., 2011).

Figura 9 - Espectros de RPE da (a) porfirina de manganês MnTBMPP 6; e, (b) porfirina de ferro, FeTBMPP 7.

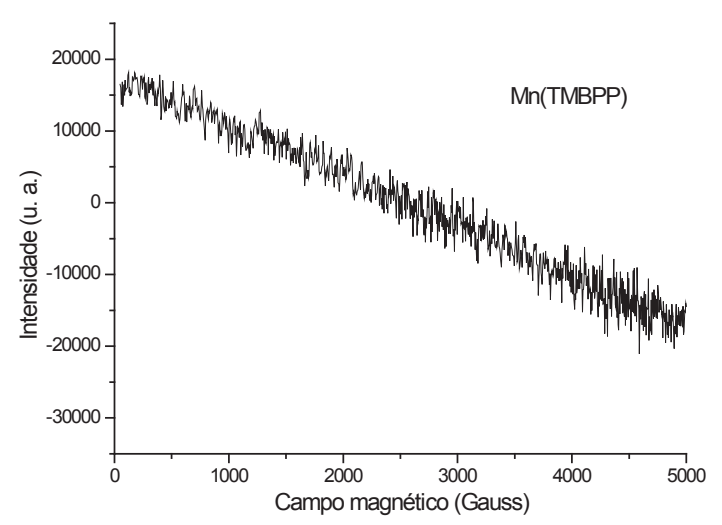

(a)

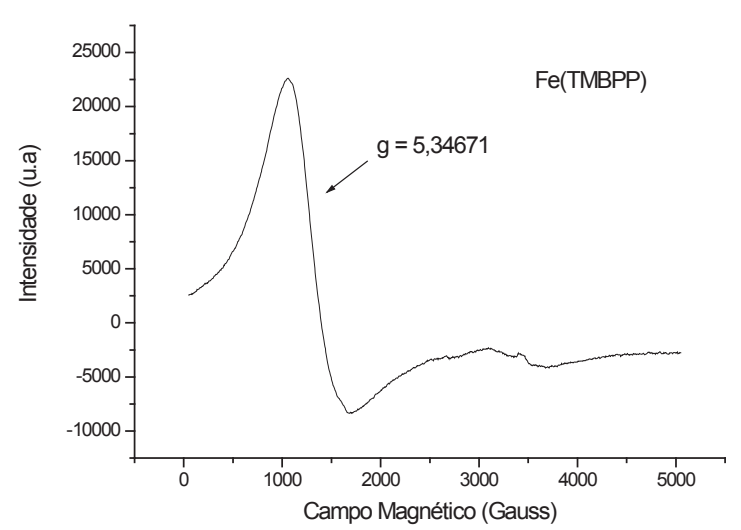

(b)

Fonte: Dados do autor. 
Neste estudo também foram preparadas e caracterizadas as metalo porfirinas de zinco e cobre, no entanto, para estudos de catálise, as de ferro e manganês são preferencialmente utilizadas (DOLPHIN; TRAYLOR; XIE, 1997; MANSUY, 1990).

\section{Conclusões}

A obtenção e caracterização de uma nova porfirina e metalo porfirinas foi atingido com êxito.

Neste trabalho foram obtidos derivados porfirínicos metalados utilizando-se a vanilina como precursor sintético, e a síntese da porfirina na forma de base livre pode ser realizada em escala multigramas utilizando o método de Adler-Longo.

O grupo $n$-butil conferiu aos compostos sintetizados 5-7 solubilidade em uma grande variedade de solventes orgânicos, tornando-os versáteis em estudos de atividade catalítica ou incorporação em filmes finos para utilização como sensores, entre outros.

\section{Agradecimentos}

Ao CNPq, a Capes, a Fundação Araucária, ao PPGQA/UEPG, ao Dequim/UEPG, ao DQ/UFPR e a Prof. ${ }^{a}$ Dr. ${ }^{a}$ Shirley Nakagaki e seu grupo do Laboratório de Bioinorgânica e Catálise (DQ/ UFPR).

\section{Referências}

ADLER, A. D.; LONGO, F. R.; FINARELLI, J. D.; GOLDMACHER, J.; ASSOUR, J.; KORSAKOFF, L. A simplified synthesis for meso-tetraphenylporphine. The Journal of Organic Chemistry, Washington, v. 32, n. 2, p. 476, 1967.

ARMAREGO, W. L. F.; PERRIN, D. D. Purification of Laboratory Chemicals. 4. ed. Oxford: ButterworthHeinemann, 2000.
BOUCHER, L. J. Manganese porphyrin complexes. III. Spectroscopy of chloroaquo complexes of several porphyrins Journal of the American Chemical Society, Easton, v. 92, n. 9, p. 2725-2730, 1970.

DOLPHIN, D.; TRAYLOR, T. G.; XIE, L. Y. Polyhaloporphyrins: unusual ligands for metals and metal-catalyzed oxidations. Accounts of Chemical Research, Washington, v. 30, n. 6, p. 251-259, 1997.

FRIEDERMANN, G. R; HALMA, M.; CASTRO, K. A. D. F.; BENEDITO, F. L.; DORO, F. G.; DRECHSEL, S. M.; MANGRICH, A. S.; ASSIS, M. D.; NAKAGAKI, S. Intermediate species generated from halogenated manganese porphyrins electrochemically and in homogeneous catalysis of alkane oxidation. Applied Catalysis A, Amsterdam, v. 308, p. 172-181, 2006.

GONSALVES, A. M. A. R.; VAREJÃO, J. M. T. B.; PEREIRA, M. M. Some new aspects related to the synthesis of mesosubstituted porphyrins. Journal of Heterocyclic Chemistry, Albuquerque, v. 28, n. 3, p. 635640, 1991.

GOUTERMAN, M. Spectra of porphyrins. Journal of Molecular Spectroscopy, New York, v. 6, p. 138-163, 1961.

JOHNSTONE, R. A. W.; NUNES, M. L. P. G.; PEREIRA, M. M.; GONSALVES, A. M. A. R.; SERRA, A. C. Improved syntheses of 5,10,15,20-tetrakisaryl- and tetrakisalkylporphyrins. Heterocycles, Sendai, v. 43, n. 7, p. 1423-1437, 1996.

KAIN, W.; SCHWEDERSKI, B. Bioinorganic Chemistry: Inorganic Elements in the Chemistry of Life: an introduction and guide. New York: John Wiley \& Sons, 1994.

KIM, J. B.; LEONARD, J. J.; LONGO, F. R. Mechanistic study of the synthesis and spectral properties of mesotetraarylporphyrins. Journal of the American Chemical Society, Easton, v. 94, n. 11, p. 3986-3992, 1972.

LINDSEY, J. S.; SCHREIMAN, I. C.; HSU, H. C.; KEARNEY, P. C.; MARGUERETTAZ,A. M. Rothemund and Adler-Longo reactions revisited: synthesis of tetraphenylporphyrins under equilibrium conditions. The Journal of Organic Chemistry, Washington, v. 52, n. 5, p. 827-836, 1987.

MANSUY, D. Biomimetic catalysts for selective oxidation in organic chemistry. Pure and Applied Chemistry, Oxford, v. 62, n. 4, p. 741-746, 1990.

MILGROM, L. R. The colours of life: an introduction to the chemistry of porphyrins and related compounds. Oxford: Oxford University Press, 1997. p. 1-20. 
NERI, C. R.; SERRA, O. A.; MAESTRIN, A. P. J.; IAMAMOTO, Y.; FERREIRA, A. G. Síntese, caracterização e estudos de transferência de energia do complexo dimérico constituído por $\mathrm{Zn}$ e Mn porfirina. Eclética Química, São Paulo, v. 27, p. 1-18, 2002.

NIAN, J. L.; MIN, L.; KONG, H. A. Syntheses and characterization of some porphyrins and metalloporphyrins. Inorganica Chimica Acta, Lausanne, v. 178 , n. 1, p. 59-65, 1990.

PRADO-MANSO, C. M. C.; VIDOTO, E. A.; VINHADO, F. S.; SACCO, H. C.; CIUFFI, K. J.; MARTINS, P. R.; FERREIRA, A. G.; LINDSAYSMITH, J. R.; NASCIMENTO, O. R.; IAMAMOTO, Y. Characterization and catalytic activity of iron (III) mono(4- $N$-methyl pyridyl)-tris(halophenyl) porphyrins in homogeneous and heterogeneous systems. Journal of Molecular Catalysis A: Chemical, v. 150, p. 251-266, 1999.

SCHIAVON, M. A.; IWAMOTO, L. S.; FERREIRA, A. G.; IAMAMOTO, Y.; ZANONI, M. V. B.; ASSIS, M. D. Synthesis and characterization of a novel series of meso(nitrophenyl) and meso(carboxyphenyl) substituted porphyrins. Journal of the Brazilian Chemical Society, v. 11, n. 5, p. 458-466, 2000.
SHAW, S. J.; SHANMUGATHASAN, S.; CLARKE, O. J.; BOYLE, R. W.; OSBORNE, A. G.; EDWARDS, C. ${ }^{13} \mathrm{C}$ NMR spectral examination of $\alpha$ - and $\beta$-carbon signal peak heights in some disubstituted arylporphyrins. Journal of Porphyrins and Phthalocyanines, Hoston, v. 5, p. 575-581, 2001.

SIMPLICIO, F. I.; MAIONCHI, F.; HIOKA, N. Terapia fotodinâmica: aspectos farmacológicos, aplicações e avanços recentes no desenvolvimento de medicamentos. Quimica Nova, São Paulo, v. 25, n. 5, p. 801-807, 2002.

SMITH, K. M. Syntheses and chemistry of porphyrins. Journal of Porphyrins and Phthalocyanines, Hoston, v. 4, p. 319-324, 2000.

UCOSKI, G. M.; CASTRO, K. A. D. F.; CIUFFI, K. J.; RICCI, G. P.; MARQUES, J. A.; NUNES, F. S.; NAKAGAKI, S. Use of iron and manganese porphyrins in solution and immobilized on silica obtained by the sol-gel process as catalyst in the oxidation of organic substrates. Applied Catalysis A: General, v. 404, p. 120128, 2011. 
